\title{
Guillain-Barré syndrome and dengue fever: report on ten new cases in Brazil
}

\author{
Síndrome de Guillain-Barré e dengue: relatório sobre dez novos casos no Brasil
}

Yara Dadalti Fragoso', Sidney Gomes², Joseph Bruno Bidin Brooks ${ }^{1}$, Andre Palma da Cunha Matta ${ }^{3}$, Heloisa Helena Ruocco ${ }^{4}$, Carlos Bernardo Tauil', Nise Alessandra de Carvalho Sousa ${ }^{6}$, Caroline Vieira Spessotto7, Talyta Grippe 5

Dear Editor,

The clinical presentation of dengue fever may vary from minimal symptoms to high fever, joint and muscle pain, headache and skin rashes, and even to hemorrhagic or shock syndromes. Neurological manifestations of this disease may come as a result of direct dengue virus invasion in the central nervous system $(\mathrm{CNS})^{1}$, and/or as neuroimmunological syndromes affecting either the CNS or the peripheral nerves'2. There is a paucity of data on cases like these, particularly regarding the discussion on therapy and prognosis. The present study reports on a series of 10 patients with Guillain-Barré syndrome (GBS) in association with dengue fever in Brazil and discusses therapeutic approaches and prognostic data on these cases. The study was approved by the Ethics Committee of Universidade Metropolitana de Santos. Data on patients with GBS in association with dengue fever were collected by neurologists from seven different Brazilian institutions in areas where dengue fever is epidemic. Only cases with complete data were included in this series.

Table. Data on patients with Guillain-Barré syndrome in association with dengue fever. All the patients had positive serum tests for dengue fever, electroneuromyography showing inflammatory peripheral polyneuropathy, and cerebrospinal fluid with protein-cell dissociation. Serum tests were negative for Zika virus and chikungunya virus.

\begin{tabular}{|c|c|c|c|c|c|c|c|}
\hline Case & Gender & Age & $\begin{array}{l}\text { Ethnic } \\
\text { origin* }\end{array}$ & $\begin{array}{c}\text { Days between DF } \\
\text { and GBS }\end{array}$ & Neurological manifestations & Treatment & Clinical outcome \\
\hline 1 & $\mathrm{~F}$ & 40 & white & 10 & $\begin{array}{c}\text { Dysphonia, dysphagia, bilateral } \\
\text { facial nerve paralysis, tetraparesis, } \\
\text { paresthesia, areflexia }\end{array}$ & Immunoglobulin & $\begin{array}{l}\text { Fully recovered } \\
\text { after } 6 \text { months }\end{array}$ \\
\hline 2 & $\mathrm{~F}$ & 42 & white & 5 & Tetraparesthesia & Immunoglobulin & $\begin{array}{l}\text { Fully recovered } \\
\text { after } 4 \text { months }\end{array}$ \\
\hline 3 & M & 50 & white & 10 & Paraparesis, paresthesia & Immunoglobulin & $\begin{array}{l}\text { Fully recovered } \\
\text { after } 1 \text { month }\end{array}$ \\
\hline 4 & M & 17 & black & 14 & Tetraparesis & Immunoglobulin & $\begin{array}{l}\text { Fully recovered } \\
\text { after } 3 \text { months }\end{array}$ \\
\hline 5 & M & 29 & white & 12 & Tetraparesis & Immunoglobulin & $\begin{array}{l}\text { Fully recovered } \\
\text { after } 3 \text { months }\end{array}$ \\
\hline 6 & $\mathrm{~F}$ & 25 & white & 7 & Tetraparesis & Immunoglobulin & $\begin{array}{l}\text { Fully recovered } \\
\text { after } 2 \text { months }\end{array}$ \\
\hline 7 & M & 40 & black & 12 & $\begin{array}{l}\text { Dysphagia, bilateral facial nerve } \\
\text { paralysis, tetraparesis, areflexia }\end{array}$ & Immunoglobulin & $\begin{array}{l}\text { Fully recovered } \\
\text { after } 25 \text { days }\end{array}$ \\
\hline 8 & M & 37 & black & 15 & Paraparesis, paresthesia & Immunoglobulin & $\begin{array}{l}\text { Fully recovered } \\
\text { after } 9 \text { days }\end{array}$ \\
\hline 9 & M & 16 & black & 14 & Tetraparesis, areflexia & Immunoglobulin & Fully recovered \\
\hline 10 & $\mathrm{~F}$ & 24 & white & 10 & Tetraparesis, tetraparesthesia & Immunoglobulin & $\begin{array}{l}\text { Fully recovered } \\
\text { after } 12 \text { months }\end{array}$ \\
\hline
\end{tabular}

${ }^{1}$ Universidade Metropolitana de Santos, Departamento de Neurologia, Santos SP, Brasil;

${ }^{2}$ Hospital Beneficência Portuguesa e Hospital Paulistano, Departamento de Neurologia, São Paulo SP, Brasil;

${ }^{3}$ Universidade Federal Fluminense, Departamento de Neurologia, Niterói RJ, Brasil;

${ }^{4}$ Faculdade de Medicina de Jundiaí, Departamento de Neurologia, Jundiaí SP, Brasil;

${ }^{5}$ Hospital de Base do Distrito Federal, Departamento de Neurologia, Brasília DF, Brasil;

${ }^{6}$ Hospital Universitário Getúlio Vargas, Departamento de Neurologia, Manaus AM, Brasil;

${ }^{7}$ Angiocopore, Departamento de Medicina Interna e Cuidado Intensivo, Santos SP, Brasil.

Correspondence: Yara Dadalti Fragoso; Departamento de Neurologia da Faculdade de Medicina da UNIMES; Rua da Constituição, 374 ; 11015 -470 Santos SP, Brasil; E-mail:yara@bsnet.com.br

Received 17 June 2016; Received in final form 01 August 2016; Accepted 24 August 2016. 
The data on 10 patients with GBS in association with dengue fever are summarized in the Table. The clinical manifestations were variable and often severe, but recovery was mostly complete and fast. Acute motor sensory axonal neuropathy was identified in all cases. All the patients were treated with a five-day pulse of immunoglobulin and responded well. However, full recovery took a variable amount of time, ranging from nine days to one year.

We highlight that GBS in association with dengue fever is a rare condition, with less than 20 cases described in detail in the literature. A recent report on three cases in New Caledonia showed findings similar to those reported here ${ }^{3}$, while fatal cases have recently been described in India ${ }^{4}$ and Pakistan $^{5}$. In Brazil, which has been an endemic region for dengue fever for over two decades, very few cases of associated GBS have been reported ${ }^{6,7,8,9}$. These authors believe that GBS in association with dengue fever may be underdiagnosed, even in endemic areas. It is a relatively benign condition that can successfully be treated with pulses of immunoglobulin if diagnosed early.

\section{References}

1. Puccioni-Sohler M, Rosadas C. Advances and new insights in the neuropathogenesis of dengue infection. Arq Neuropsiquiatr. 2015;73(8):698-703. doi:10.1590/0004-282X20150074

2. Verma R, Sahu R, Holla V. Neurological manifestations of dengue infection: a review. J Neurol Sci. 2014;346(1-2):26-34. doi:10.1016/j.jns.2014.08.044

3. Simon O, Billot S, Guyon D, Daures M, Descloux E, Gourinat AC, et al. Early Guillain-Barré Syndrome associated with acute dengue fever. J Clin Virol. 2016;77:29-31. doi:10.1016/j.jcv.2016.01.016

4. Mishra V, Harbada R.Fatal Guillain-Barre Syndrome (GBS) in dengue. J Assoc Physicians India. 2015;63:94-6.

5. Assir MZ, Ahmad HI, Masood MA, Kamran U, Yusuf NW. Deaths due to dengue fever at a tertiary care hospital in Lahore, Pakistan. Scand J Infect Dis. 2014;46(4):303-9. doi:10.3109/00365548.2013.877155
6. Gonçalves E. Acute inflammatory demyelinating polyradiculoneuropathy (Guillain-Barré syndrome) following dengue fever. Rev Inst Med Trop Sao Paulo. 2011;53(4):223-5. doi:10.1590/S0036-46652011000400009

7. Puccioni-Sohler M, Soares CN, Papais-Alvarenga R, Castro MJ, Faria LC, Peralta JM. Neurologic dengue manifestations associated with intrathecal specific immune response. Neurology. 2009;73(17):1413-7. doi:10.1212/WNL.0b013e3181bd8258

8. Santos NQ, Azoubel AC, Lopes AA, Costa G, Bacellar A. Guillain-Barré syndrome in the course of dengue: case report. Arq Neuropsiquiatr. 2004;62(1):144-6. doi:10.1590/S0004-282X2004000100025

9. Ferreira ML, Cavalcanti CG, Coelho CA, Mesquita SD. [Neurological manifestations of dengue: study of 41 cases]. Arq Neuropsiquiatr. 2005;63(2B):488-93. Portuguese. doi:10.1590/S0004-282X2005000300023 